\title{
Sitzung vom 8. März 1875.
}

Vorsitzender: Hr. C. Scheibler, Vicepräsident.

Das Protocoll der letzten Sitzung wird genehmigt.

Gewählt werden:

1) als einheimische Mitglieder:

die Herren :

K. O. Čech, Dr. phil.,

Emmerling, stud. phil.,

Leupold, stud. phil,

Universitätslaboratorium

Georgenstr. 35;

2) als auswärtige Mitglieder:

die Herren:

Dr. med. Franz Hofmeister, Assistent am medic.-chem.

Laborat. der Universität Prag,

Dr. G. Th. Gerlach in Kalk bei Cöln,

Otto Gluge, Assistent am technischen Laborat. des Polytechnicums zu Aachen,

Ley, Docent am landwirthsch. Institut zu Nova-Alexandria, Königr. Polen,

Dr. P. T. Cleve, Professor an der Universităt Upsala,

Fr. Gantler, Assistent in Hohenbeim bei Stuttgart,

E. Jobelmann, Apotheker in Altenbruch bei Stade,

A. Lauber, Chemiker der Centralstelle für Gewerbe und Handel in Stuttgart,

C. Stahl, stud. chem.,

Franz Gaess, stud. chem., Polytechnicum in Stuttgart,

Emil Wahl, Assistent,

Robert Ebert,

Jonze Tibiriçá Piratininza,

Jacob Czarnowsk.y,

Joseph Walzyk,

Goesta Fries, .

Carl Schelnberg,

A. Ennes de Souza,

Wladyslaus Au,

Hans S chü tz.

Universitätslaboratorium

zu Zürich.

Borichte d. D. Chem. Genollachaft. Jahrg. VIIl, 
In der Sitzung vom 21. Februar sind folgende Mitglieder gewählt worden, deren Namen hiermit nachgetragen werden:

1) als einheimische Mitglieder:

die Herren:

D awson, Universitätslaboratorium, Georgenstr. 35,

Köhler, stud. chem., Fürstenstr. 13 III, Reimann, stud. chem., gr. Hamburgerstr. 25 III,

Alker, stud. chem., Gypsstr. 12 part.,

Plath, stud. chem., Bartelstr. 4 I;

2) als auswärtige Mitglieder:

die Herren:

Julius Laupheimer, Chemiker der Düngerfabrik v. Michel,

Lederer \& Co. in Ludwigshafen,

Johann Kross, Apotheker in Husum,

Paul Behrend, stud. chem., Waisenbausstr. 38 in Leipzig, Edouard Kopp,

Stanislaus Wrzesinsky,

Ferdinand Girtanner,

Karl Kussmaul,

Ladislaus Klobukowsky,

Laboratorium des Polytechnicums in Zürich,

Prof. Th. Haas in Stuttgart, pr. Adr. Badische Anilin- und

Sodafabrik in Ludwigshafen a./Rh.,

F. W. Felten, Rüsselbeim a./Main bei Mainz,

Eugen Fischer, Privatdocent. u. Assist. a. chem.-technol.

Hans Vogel, Lab. des Polytechnicums zu Stuttgart,

Dr. Isidor Walz, Consulting Chemist, 18. Exchange Place,

New-York, U. S. A.,

Dr. Walter Nägeli, Augustenstr. 8 in München,

Georg Ammon, Assist. a. d. Veterinärschule in München,

Dr. Wilhelm Miller, Assistent am chemischen Institut des

Polytechnicums in München,

Dr. Carl Helbig,

Adolf Kayser,

Jos. Brand,

G. Strokirk,

Max Wassermann,

J. Braunstein,

Emil Knudsen,

Carl Methner,

Jos. Jais,

Heinrich von Ziegesar,

Oppeln von Bronikowsky,

Adolf $\mathrm{Ott}$,

Chemisehes Laboratorium des Polytechnicums in München, 
Jos. C. A. Brunner,

Moritz Sachs,

Albert Kehlstadt, Bernhard Althaus, Joseph Brunbauer, Michael Fischer,

H. Kast,

M. G. Kressner,

Paul Silber,

Georg Rietschel,

Chemisches Laboratorium

des Polytechnicums

in. München.

Für die Bibliothek sind eingegangen:

Als Geschenk:

Bericht über die Entwickelung der chemischen Industrie, während des letzten Jahrzehnts, im Verein mit Freunden und Fachgenossen erstattet von A. W. Hofmann. 1. Heft. Braunschweig 1875.

Eichhorn: Einige Beiträge zu den Absorptions-Erscheinungen in den Ackererden. (Vom Verf.)

Bolletino della societ̀̀ Adriatica di scienze naturali in Trieste. No. 1. 1875.

Polytechnisches Notizblatt. No. 4, 5.

Repertorium der Naturwissenschaften. Monatl. Uebersicht der neuesten Arbeiten auf dem Gebiete der Naturwissenschaften. Herausgegeb. von der Redaction des Naturforscher. 1. Jahrg. No. 1, 2. (Von der Verlagshandlung.)

Der Naturforscher. Jahrg. 1875, Jan. u. Febr. (Von der Verlagshandlung.)

Ferner folgende Zeitschriften im Austausch:

J. Liebig's Annalen der Chemie. Bd. 176, Heft 1.

Chemisches Centralblatt. No. $8,9$.

Deutsche Industriezeitung. No. 9.

Landwirthschaftliche Jahrbücher. Bd. IV. Heft 1.

Monatsberichte der Kgl. Preuss. Acad. der Wissenschaften zu Berlin. Aug., Sept., Oct. 1874.

Verhandlungen der K. K. geolog. Reichsanstalt. No. 2.

Vierteljahrsschrift der naturforschenden Gesellschaft zu Zürich. XVIII. Jahrg. (1873).

Heft $1-4$.

Bulletin de la Société chimique de Paris. No. 5.

Revue hebdomadaire de Chimie. No. 4-6.

Revue scientifique. No. 35, 36.

Maandblad voor Natuurwetenschappen. No. 3.

Durch Kauf:

Comptes rendus. No. 7-9.

\section{Mittheilungen.}

\section{C. Göpner: Ueber das sogenannte Chlorhydrat.}

(Eingegangen am 2. März; verles. in der Sitzung von Hrn. Oppenbeim.)

In den meisten der neueren anorganischen Lehrbücher wird angegeben, dass das Chlorhydrat $\mathrm{Cl}_{2}+10 \mathrm{H}_{2} \mathrm{O}$ auch als $\mathrm{HOCl}+\mathrm{HCl}$ $+9 \mathrm{H}_{2} \mathrm{O}$ angesehen werden könne. Gründe für diese Auffassungs- 